\title{
Caso clínico
}

\section{Hiperamonemia como causa de diálisis no renal}

\author{
Benjamín Wancjer Meid ${ }^{(D)}{ }^{1}$, Alejandra Molano Triviño ${ }^{(D)}{ }^{1}$, Samuel Yucuma \\ Gutiérrez ${ }^{(D)}{ }^{1}$ y Carlos Mauricio Martínez Montalvo ${ }^{D} \square^{2}$ \\ ${ }^{1}$ Departamento de Nefrología, Escuela de Medicina y Ciencias de la Salud, Universidad del Rosario, Bogotá D.C., \\ Colombia. \\ ${ }^{2}$ Medicina Interna, Escuela de Medicina y Ciencias de la Salud, Universidad del Rosario, Bogotá D.C., Colombia.
}

Cómo citar: Wancjer Meid B, Molano Triviño A, Yucuma Gutiérrez S, Martínez Montalvo CM. Hiperamonemia como causa de diálisis no renal. Rev. Colomb. Nefrol. 2021; 8(1), e385. https:/ doi.org/10.22265/acnef.8.1.385

Recibido:

$14 / \mathrm{Feb} / 2020$

Aceptado:

28/Sep/2020

Publicado:

$11 /$ Nov/2020

\section{Resumen}

La hiperamonemia es una condición frecuente en pacientes cirróticos y en el contexto de una causa no cirrótica se relaciona con aumento en la producción de amonio o alteración en su eliminación. La presentación clínica de esta condición es inespecífica: va desde alteración del comportamiento hasta estado de coma, siendo una de las causas de diálisis no renal por determinados valores o refractariedad a manejo médico.

Se presenta el caso de una mujer de 35 años, quien ingresó al servicio de urgencias con alteración del estado de conciencia, niveles de amonio elevados y masa hipervascular en hígado. Los estudios de aminoácidos limitantes del ciclo de la urea y ácido orótico urinario fueron normales y la biopsia de la lesión hepática reportó carcinoma hepático fibrolamelar, al cual se atribuyó la presencia de shunt portosistémico que causaba la hiperamonemia. Se indicó diálisis por persistencia de elevaciones de amonio y poca respuesta al tratamiento médico. Dada la evolución de la paciente, fue necesario reiniciar la terapia reemplazo renal por reaparición de estado encefalopático al suspender la misma. Esta terapia se mantuvo hasta el trasplante hepático que se realizó como tratamiento del carcinoma, con posterior estabilización de niveles de amonio y suspensión de la diálisis.

Palabras clave: cáncer hepático, hiperamonemia, encefalopatía, diálisis.

$\square$ Correspondencia: Carlos Martínez, Escuela de Medicina y Ciencias de la Salud, Universidad del Rosario, Sede Quinta Mutis, Carrera 24 No. 63C-69, Bogotá D.C., Colombia. Correo-e: carlosm.martinez@urosario.edu.co 


\title{
Hyperammonemia as a cause of non-renal dialysis
}

\begin{abstract}
Hyperammonemia is a common condition in cirrhotic patients. In the context of a noncirrhotic cause, this is related to the increase in its production of ammonium or alteration in its elimination. The clinical presentation is nonspecific, from the alteration of the behavior to the coma, being one of the causes of non-renal dialysis due to certain values or refractoriness to medical management. The case of a 35- year-old woman is presented, who is admitted to the emergency department with altered state of consciousness, elevated ammonium levels and hypervascular mass in the liver. Biopsy of the liver lesion reports fibrolamellar liver carcinoma. The presence of a portosystemic shunt that causes hyperammonemia is attributed to this pathology. Dialysis is indicated by persistence of ammonium elevations with little response to medical treatment. In its evolution, it required a restart of renal replacement due to a reappearance of the encephalopathic state when it was suspended. This therapy is maintained until liver transplantation performed as a carcinoma treatment, with subsequent stabilization of ammonium levels and dialysis suspension.
\end{abstract}

Keywords: liver neoplasm, ammonia, encephalopathy, dialysis.

\section{Introducción}

La hiperamonemia como causa de encefalopatía es una condición usualmente relacionada con cirrosis hepática y un marcador de pobre pronóstico [1]. La hiperamonemia no cirrótica es una entidad rara, por lo cual requiere una confirmación de la muestra para evitar falsos positivos [2], además es una de las indicaciones de diálisis no renal cuando se observan valores de amonio elevados en forma persistente y con refractariedad al manejo médico [3].

Las causas de hiperamonemia se pueden clasificar en dos grupos: en el primero se encuentran las causas relacionadas con el incremento de la producción de amonio, en las que se incluye infecciones, desórdenes hematooncológicos, catabolismo aumentado, entre otros, y en el segundo se incluyen las condiciones relacionadas con disminución en la eliminación del amonio, tales como errores innatos del metabolismo de la urea y shunts portosistémico e inducidos por medicamentos [4]. Del mismo modo, diversos tumores han sido relacionados con hiperamonemia, entre los que se encuentra el carcinoma hepático fibrolamelar (FL-CHC), un tumor hepático primario no relacionado con enfermedad hepática previa o cirrosis que es típico de personas jóvenes y causa hasta un $9 \%$ de los carcinomas hepatocelulares [5]. 
A continuación, se reporta el caso raro de una mujer de 35 años con alteración del estado de conciencia asociado a ictericia e hiperamonemia severa con hallazgos de masa hepática, a quien se le indicó diálisis hasta resolución de la causa luego de un trasplante hepático.

\section{Presentación del caso}

Paciente femenina de 35 años de edad con antecedente de masa abdominal en estudio y parto sin complicaciones 3 meses atrás, quien ingresó al servicio de urgencias por cuadro clínico consistente en alteración del estado de conciencia dado por episodio convulsivo. Al ingreso, la paciente se encontraba con estabilidad hemodinámica, estuporosa, afebril con ictericia y hepatomegalia. Dentro de los paraclínicos se evidenció hiperbilirrubinemia de predominio directo, elevación de transaminasas e hiperamonemia (Tabla 1). Sus familiares refirieron historia clínica negativa para infecciones y consumo de alcohol, medicamentos o tóxicos, pero mencionaron la presencia de una masa abdominal que apareció nueve meses

\section{Se llevó a cabo un estudio de líquido cefalorraquídeo que no reportó alteraciones}

antes. Debido al deterioro del estado de conciencia, se decidió asegurar vía aérea con intubación orotraqueal e iniciar manejo en unidad de cuidado intensivo (UCI) con equipo multidisciplinario y lactulosa, rifaximina, Larginina y L- aspartato (LOLA).

Dada la evaluación de la paciente, se ordenaron otros exámenes como detección de virus de hepatitis B y C y virus de inmunodeficiencia humana, perfil de autoinmunidad y marcadores tumorales como alfafetoproteína, siendo todos negativos o en rango normal (Tabla 1).

Respecto a las ayudas imagenológicas, se realizó una tomografía simple de cráneo que evidenció lesiones focales hipodensas en sustancia blanca periventricular de aspecto inespecífico sin efecto de masa (Figura 1); dado que estos hallazgos no descartaron neuroinfección, se llevó a cabo un estudio de líquido cefalorraquídeo que no reportó alteraciones. Posteriormente, se ordenó una resonancia magnética nuclear de abdomen que detectó hígado aumentado de tamaño y disminución de la intensidad de señal de manera difusa por infiltración de grasa hepática; además, en el segmento VIII se observó lesión focal hepática hipervascular de contornos lobulados, forma irregular y diámetro de $13 \times 11 \times 13 \mathrm{~cm}$, con compresión de venas suprahepáticas, vena cava inferior y vena porta intrahepática izquierda con compresión de la vía biliar intrahepática (Figura 2). Dado el hallazgo de masa hepática, se decidió tomar biopsia de la lesión, cuyo reporte histopatológico fue compatible con FL-CHC (Figura 3). 
Tabla 1. Resultados de laboratorios

\begin{tabular}{|l|c|}
\hline \multicolumn{1}{|c|}{ Paraclínicos } & Resultados \\
\hline Leucocitos (/uL) & 10.940 \\
\hline Hemoglobina (g/dL) & 15 \\
\hline Plaquetas (/uL) & 544.000 \\
\hline Bilirrubina (mg/dL) & 2,73 \\
\hline Bilirrubina directa & 264 \\
\hline AST (IU/L) & 261 \\
\hline ALT (IU/L) & 477 \\
\hline FA (IU/L) & $13,1 / 1,2$ \\
\hline PT (sec)/INR & 18,2 \\
\hline BUN (mg/dL) & 0,51 \\
\hline Creatinina(mg/dL) & 86 \\
\hline VSG & 2,2 \\
\hline Albumina & 4,6 \\
\hline Proteinas totales & 1,16 \\
\hline AFP & $<7,0(0-16,9)$ \\
\hline Anticuerpos anticitrulinicos & $18(13-128)$ \\
\hline Arginina (nmol/ml) & $68(16-129)$ \\
\hline Ornitina (nmol/ml) & $9(1-33)$ \\
\hline Citrulina (nmol/ml) & $0,5(0,4-5,1)$ \\
\hline AOU (mmol/mol creatinine) & 595,7 \\
\hline Amonio $(\mu \mathrm{g} / \mathrm{dL})$ & \\
\hline
\end{tabular}

ALT: alanino aminotransferasa; AST: aspartato aminotransferasa; FA: fosfatasa alcalina; PT: tiempo ptrotrombina; BUN: nitrógeno ureico en sangre; VSG: volumen sedimentación globular; AFP: alfa feto proteína; AOU: ácido orótico urinario.

Fuente: elaboración propia.

Durante la estancia en la UCI, la hiperamonemia persistió y no hubo mejoría del estado de conciencia, por lo cual la paciente fue evaluada en conjunto por los servicios de nefrología y genética, quienes decidieron solicitar nuevos estudios para descartar defectos de metabolismo de la urea, encontrando aminoácidos limitantes del ciclo de la urea normales y niveles de ácido orótico urinario normales (Tabla 1).

Debido a la persistencia de los valores elevados de amonio ( $>500 \mu \mathrm{g} / \mathrm{dL})$ a pesar de manejo médico, se indicó terapia de reemplazo renal en modalidad hemodiálisis continua, con lo que se logró mejoría del estado de encefalopatía y valores de amonio $<100 \mu \mathrm{g} / \mathrm{dL}$; esta terapia se suspendió al cuarto día gracias a los buenos resultados. Dos días después de la suspensión de la diálisis se observó reincidencia de síntomas de encefalopatía con nuevos valores de amonio en $320 \mu \mathrm{g} / \mathrm{dL}$, por lo cual se decidió reinstaurarla de manera permanente hasta esclarecer diagnóstico. 

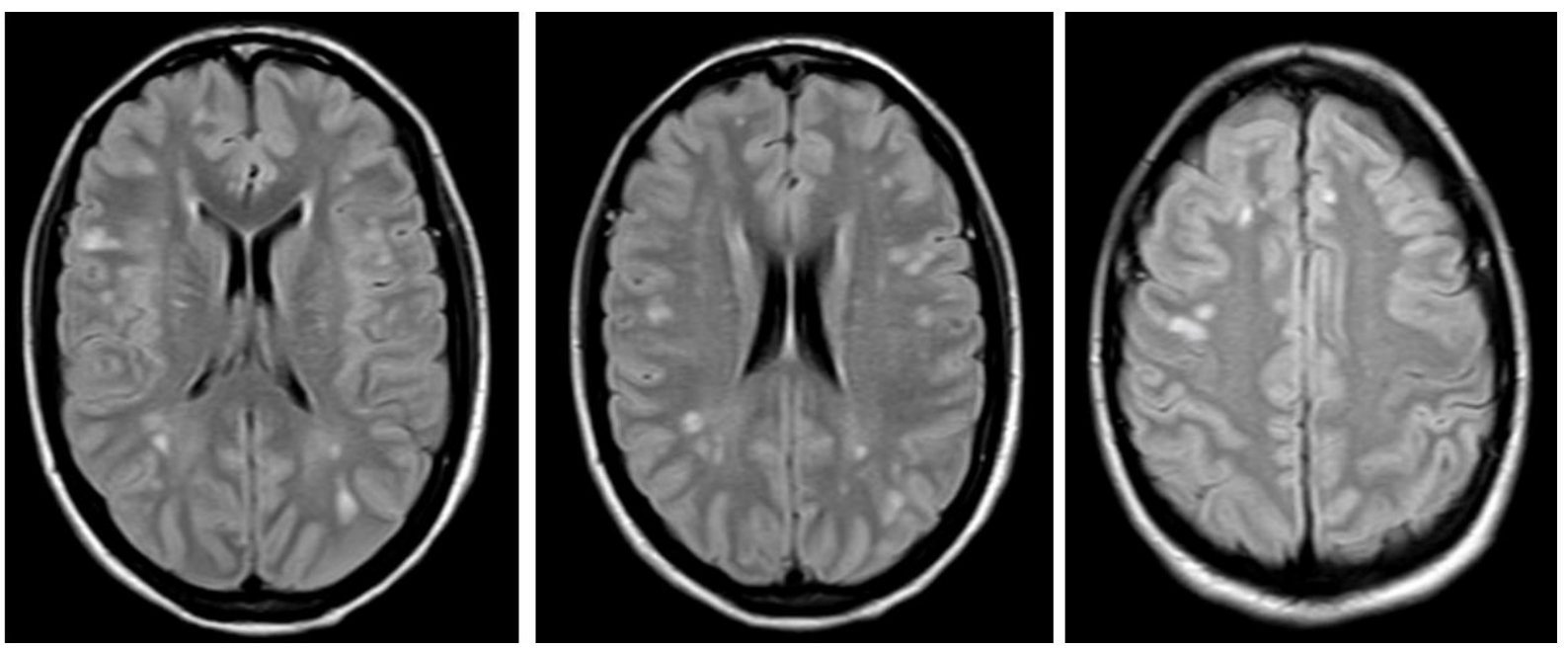

Figura 1. Tomografía simple de cráneo en paciente con hiperamonemia y carcinoma fibrolamelar

Fuente: documento obtenido durante la realización del estudio.

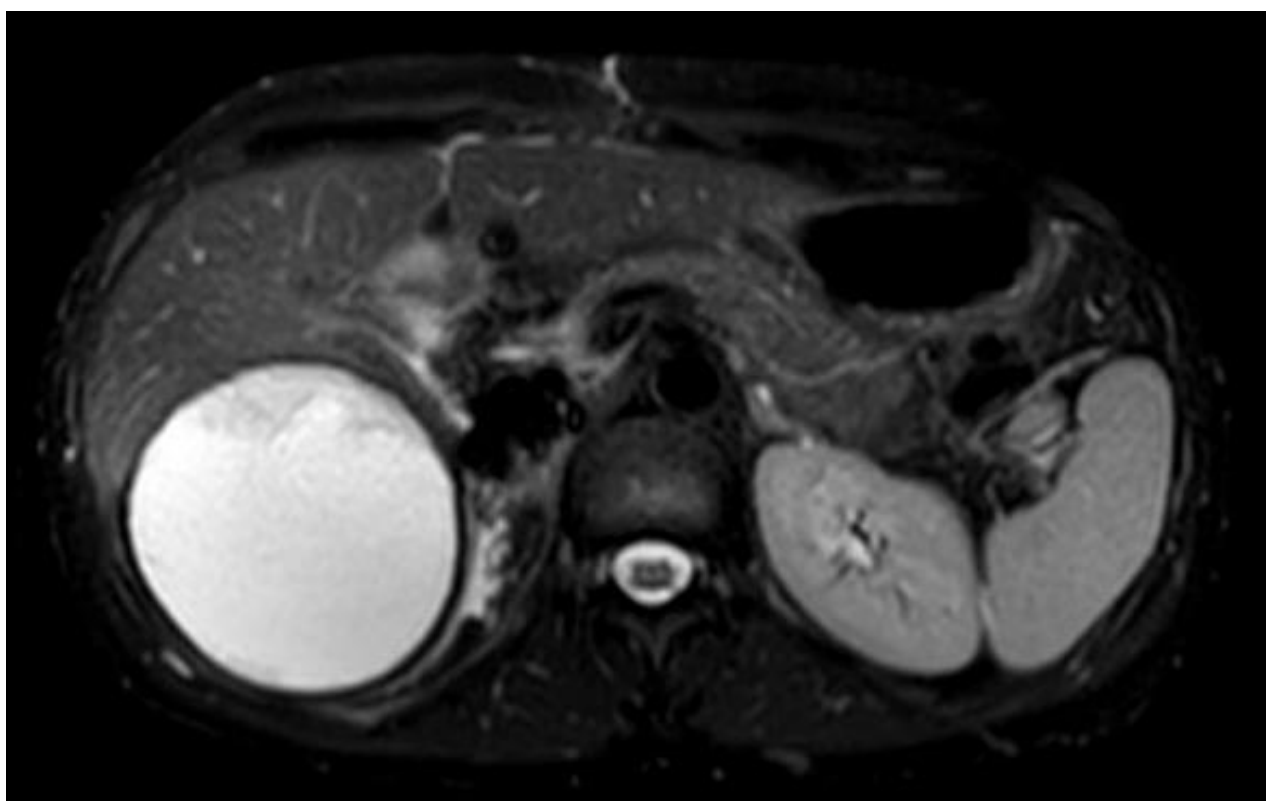

Figura 2. Resonancia magnética de abdomen con hiperamonemia y carcinoma fibrolamelar

Fuente: documento obtenido durante la realización del estudio.

En conjunto con hepatología y teniendo en cuenta los hallazgos imagenológicos, histopatológicos y paraclínicos, se llegó a la conclusión de que la paciente era candidata a trasplante hepático. La mujer continuó en diálisis hasta que recibió el trasplante, a partir de lo cual sus niveles de amonio se estabilizaron y la terapia de reemplazo renal fue suspendida. Debido a lo anterior, y al descartar errores innatos del ciclo de la urea, se atribuyó como causa de la hiperamonemia de origen no cirrótico al shunt portosistémico por masa hepática compatible 


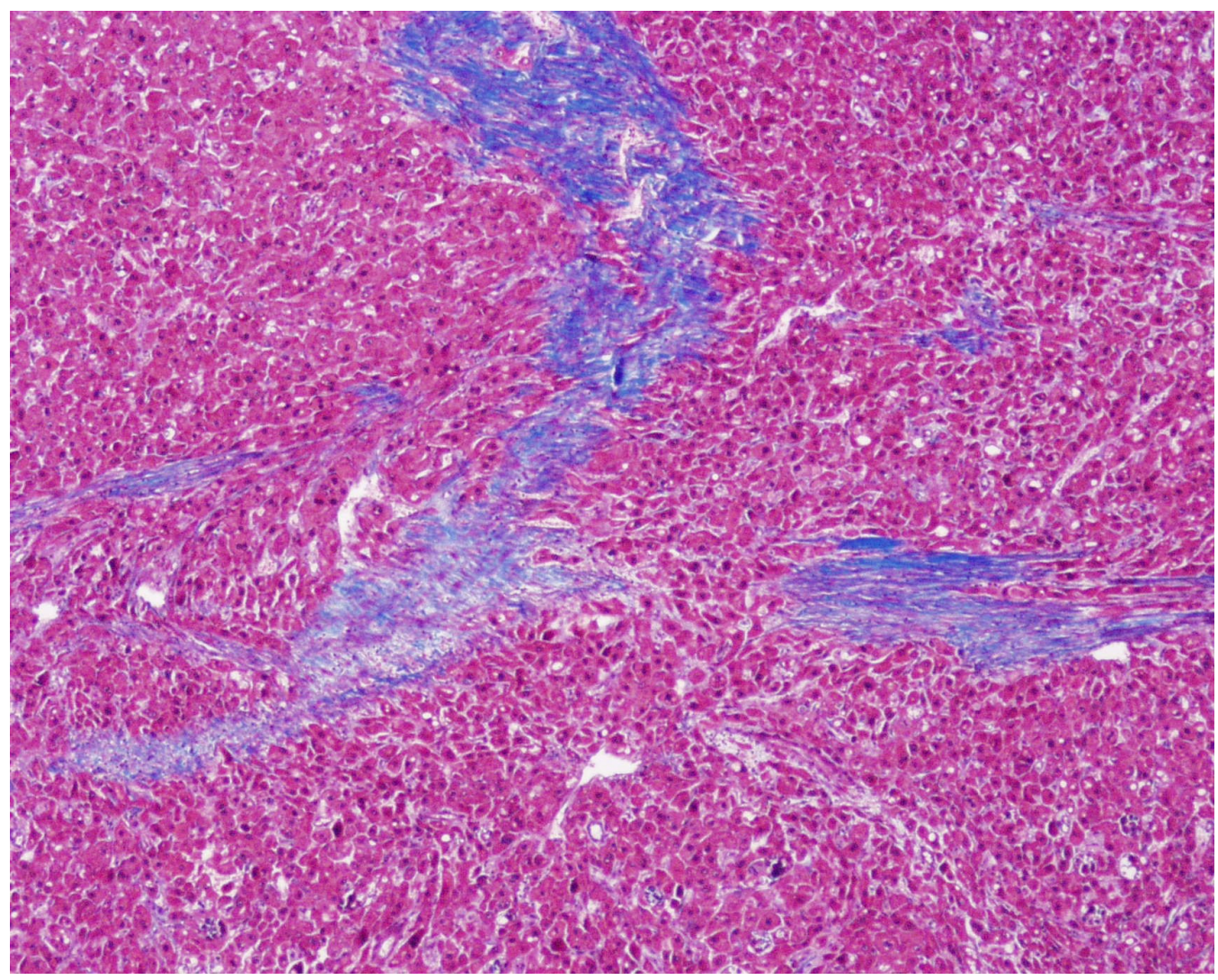

Figura 3. Biopsia hepática de carcinoma fibrolamelar

Fuente: documento obtenido durante la realización del estudio.

con FL-CHC, esto gracias a la respuesta satisfactoria de corrección de hiperamonemia luego del trasplante hepático, constituyendo la diálisis una indicación infrecuente de causa no renal.

\section{Discusión}

El amonio es una molécula orgánica correspondiente a la forma catiónica del amoniaco, además es un derivado de la urea procedente de la dieta y digestión de las proteínas; este componente es conducido por la circulación portal hasta el hígado, en donde sufre un proceso metabólico de transaminación y desaminación oxidativa para posteriormente ser excretado por el riñón [2,6]. Cuando se presenta acumulación de amonio, ya sea por mayor producción o por defecto en su eliminación, en el sistema nervioso central se ha evidenciado una toxicidad por acumulación de glutamina, proceso dado por inhibición de una enzima llamada glutaminasa [7].

Los efectos clínicos de la elevación del amonio tienen un amplio espectro de presentación, e incluyen cambios del sueño, confusión, letargia, cambios de personalidad, confusión, convulsiones y estado de coma. Por lo antes mencionado, es de vital importancia identificar 
de manera temprana la hiperamonemia para así evitar posibles noxas cerebrales que llevan consigo alteración de la homeostasis del agua cerebral y pueden producir cambios metabólicos, edema cerebral y hasta hipertensión endocraneal [8]. En el enfoque inicial de un paciente con alteración del estado de conciencia se deben descartar mimetizadores como el síndrome de Wernicke-Korsakoff, las alteraciones del sodio, la hipoglicemia, la alcalosis metabólica, el hematoma subdural crónico y el estatus epiléptico no convulsivo [1].

La paciente reportada debutó con síndrome convulsivo asociado a niveles elevados de amonio y no presentaba antecedentes de consumo crónico de alcohol ni estigmas de hepatopatía crónica o electrolitos, además su perfil metabólico era normal; sin embargo, llamaron la atención los cambios estructurales a nivel de tomografía cerebral concordantes con los cambios fisiopatológicos que ejerce el amonio en el cerebro.

En el abordaje diagnóstico de un paciente con encefalopatía sin patología hepática previa el nivel de amonio elevado debe considerarse la posibilidad de alteración en el ciclo de la urea, en el cual su abordaje diagnóstico debe hacerse con medición de niveles de aminoácidos séricos, aminoácidos urinarios, ácido orótico urinario y ácidos orgánicos urinarios [5,9]. En la paciente reportada los test realizados para diagnóstico de posibles errores innatos relacionados con el ciclo de la urea fueron negativos, por lo cual el diagnostico se tornó más difícil.

Como ya se mencionó, las etiologías de hiperamonemia de origen no cirrótico se clasifican en dos grupos, uno en el que se incluyen las causas relacionadas con el incremento de la producción de amonio: infección por bacterias productoras de urea (Proteus mirablis, Klebsiella species, Escherichia coli, Morganella morganii, Providencia rettgeri, diphtheroids, Mycobacterium genavense, Herpes simplex), desordenes hematooncológicos, quimioterapia, trasplante de órganos, aumento de consumo de proteínas o aumento en su catabolismo como es el caso en ejercicio intenso, trauma, convulsiones, La paciente reportada debutó con síndrome convulsivo asociado a niveles elevados de amonio sangrado gastrointestinal, uso de esteroides y nutrición parenteral, y otro en el que se incluyen las causas relacionadas con la disminución en la eliminación del amonio: ureterosigmoidostomía, shunt portosistémicos, medicamentos (ácido valproico, carbamazepina, rivabirin y salicilatos) y errores innatos del metabolismo [9]. En el caso reportado se descartaron uno a uno los diagnósticos, quedando la opción de un shunt portosistémico. 
Las causas de un shunt portosistémico pueden estar relacionadas con su ubicación intrahepática o extrahepática, pero en su mayoría son congénitas con manifestaciones tempranas. Otras causas incluyen lesión hepática hipervascular con o sin efecto oclusivo venoso, para lo cual la biopsia hepática en el abordaje diagnóstico descarta cirrosis, esquistosomiasis o granulomas [9]. El FL-CHC es un tipo histológico del carcinoma hepatocelular (CHC) que representa el $1 \%$ de tumores primarios hepáticos, con incidencia de 0,02 casos por cada 100.000 habitantes [10], y es causado por la mutación DNAJB1-PRKACA en el cromosoma 19 con delección de 8 genes, siendo los más comunes MUC4 (asociado a otros tumores gastrointestinales) y GOLCA6L2. El mecanismo de tumorigénesis en el FL-CHC ha sido un misterio desde su identificación inicial en 1956; sin embargo, se han logrado avances importantes tras el descubrimiento de la mutación DNA JB1-PRKACA hecho por Honeyman et al. [11] en 2014. La prevalencia de esta mutación en prácticamente todas las muestras de FL-HCC, junto con un genoma relativamente estable, sugiere fuertemente que la proteína de fusión es un impulsor primario de este tipo de cáncer [10]. Su presencia es mayor en adultos jóvenes con edad media de presentación a los 25 años, sin diferencia de género y con marcadores tumorales negativos; además, es responsable de hasta el $9 \%$ de los tumores hepáticos con compromiso del $66 \%$ del lóbulo hepático izquierdo y con descripción de asociación con hiperamonemia $[5,12,13]$.

En el proceso de entender la fisiopatología de la hiperamonemia, el FL-HCC se relaciona con el efecto de una lesión hepática hipervascular grande con presencia de shunt que disminuye el aclaramiento de productos nitrogenados $[8,12]$. En la presentación del caso reportado se observa una masa con gran ocupación del lóbulo hepático derecho y presencia de oclusión venosa como causa de hiperamonemia debido a shunt portosistémico, lo cual se evidenció al descartarse las otras etiologías y al evidenciarse la mejoría luego del trasplante hepático.

Dentro de las terapias para hiperamonemia se incluye tratamiento de presión intracraneal con manitol, hiperventilación e inducción de hipotermia; además, para el control de la producción endógena de amonio se usa lactulosa, dieta, antibióticos y limitación de la actividad muscular, y para su eliminación se emplea diálisis, benzoato de sodio y LOLA, suplemento de sustratos de ciclo de la urea con arginina y L- carnitina, y, por última, instancia el trasplante hepático $[9,13]$. La hiperamonemia es una de las indicaciones de diálisis no renal, y puede ser usada como de primera línea cuando los niveles de amonio son $>500 \mu \mathrm{g} / \mathrm{dL}$, pero también puede ser utilizada en valores entre $250 \mu \mathrm{g} / \mathrm{dL}$ y $500 \mu \mathrm{g} / \mathrm{dL}$ si la terapia médica no está disponible o si el paciente presenta refractariedad al mismo, pudiendo ser suspendida cuando se 
alcancen valores $<200 \mu \mathrm{g} / \mathrm{dL}$. Este tratamiento puede ser reforzado con la administración de arginina [5].

\section{Conclusiones}

Se presenta un caso raro de un FL-CHC que generó hiperamonemia severa asociada a encefalopatía. En el primer abordaje se realizaron estudios metabólicos y test de errores innatos de ciclo de urea que resultaron normales. Posteriormente se documentó la presencia de lesión hipervascular que condicionaba la compresión venosa llevando a un shunt portosistémico responsable de la refractariedad al manejo médico, el cual se resolvió con diálisis y tratamiento quirúrgico consistente en trasplante hepático.

\section{Consideraciones éticas}

Los autores declaran que los procedimientos seguidos se realizaron conforme a las normas éticas del comité de experimentación humana responsable y de acuerdo con lo establecido por la Asociación Médica Mundial en la Declaración de Helsinki; que han seguido los protocolos de su centro de trabajo sobre la publicación de datos de pacientes, y que han obtenido el consentimiento informado de los pacientes y/o sujetos referidos en el artículo.

\section{Conflicto de intereses}

Ninguno declarado por los autores.

\section{Financiación}

Ninguna declarada por los autores.

\section{Contribución de los autores}

Todos los autores contribuyeron en la conceptualización, curaduría de los datos, investigación, escritura (manuscrito original) y escritura (revisión y edición) del artículo.

\section{Referencias}

[1] Allampati S, Mullen KD. Nomenclature and definition of hepatic encephalopathy - An update. Clin Liver Dis (Hoboken). 2015 Apr 21;5(3):68- 70. https://doi.org/10.1002/cld.449 个Ver página 2,7 
[2] Téllez-Villajos L, Aicart-Ramos M, Moreira-Vicente V. Hiperamonemia en pacientes adultos sin cirrosis. Med Clin (Barc). 2013;141(11):494-500. https:/dx.doi.org/10.1016/j.medcli. 2013.04.040 个Ver página 2, 6

[3] Role of Dialysis in Acute Toxic Encephalopathy Secondary to Hyperammonemia. Am J Kidney Dis. 2017;69(4):A69. https://dx.doi.org/10.1053/j.ajkd.2017.02.217个Ver página 2

[4] Laish I, Ben-Ari Z. Noncirrhotic hyperammonaemic encephalopathy. Liver Int. 2011;31(9):1259-70. https://dx.doi.org/10.1111/j.1478-3231.2011.02550.x 个Ver página 2

[5] Chapuy CI, Sahai I, Sharma R, Zhu AX, Kozyreva ON. Hyperammonemic Encephalopathy Associated With Fibrolamellar Hepatocellular Carcinoma: Case Report, Literature Review, and Proposed Treatment Algorithm. Oncologist. 2016;21(4):514-20. https://x.doi. org/10.1634/theoncologist.2015-0267 个Ver página 2, 7, 8, 9

[6] Hawkes ND, Thomas GA, Jurewicz A, Williams OM, Hillier CE, McQueen IN, et al. Nonhepatic hyperammonaemia: A potentially reversible cause of encephalopathy. Postgrad Med https:/dx.doi.org/10.1136/pmj.77.913.717个Ver página6

[7] Brusilow SW, Koehler RC, Traystman RJ, Cooper AJL. Astrocyte Glutamine Synthetase: Importance in Hyperammonemic Syndromes and Potential Target for Therapy. Neurotherapeutics. 2010;7(4):452-70. https:/dx.doi.org/10.1016/j.nurt.2010.05.015 个Ver página 6

[8] Surjan RC, Dos Santos ES, Basseres T, Makdissi FF, Machado MA. A Proposed Physiopathological Pathway to Hyperammonemic Encephalopathy in a Non-Cirrhotic Patient with Fibrolamellar Hepatocellular Carcinoma without Ornithine Transcarbamylase (OTC) Mutation. Am J Case Rep. 2017;18:234- 41. https:/dx.doi.org/10.12659/ajcr.901682 个Ver página 7, 8

[9] Summar ML, Mew NA. Inborn Errors of Metabolism with Hyperammonemia: Urea Cycle Defects and Related Disorders. Pediatr Clin North Am. 2018;65(2):231-46. https://x.doi.org/ 10.1016/j.pcl.2017.11.004 个Ver página 7, 8

[10] Riggle KM, Turnham R, Scott JD, Yeung RS, Riehle KJ. Fibrolamellar Hepatocellular Carcinoma: Mechanistic Distinction From Adult Hepatocellular Carcinoma. Pediatr Blood Cancer. 2016;63(7):1163-7. https://dx.doi.org/10.1002/pbc.25970 个Ver página 8

[11] Honeyman JN, Simon EP, Robine N, Chiaroni-Clarke R, Darcy DG, Lim IIP, et al. Detection of a recurrent DNAJB1-PRKACA chimeric transcript in fibrolamellar hepatocellular carcinoma. Science. 2014;343(6174):1010-4. https:/dx.doi.org/10.1126/science.1249484个Ver página 8 
[12] Sethi S, Tageja N, Singh J, Arabi H, Dave M, Badheka A, et al. Hyperammonemic encephalopathy: A rare presentation of fibrolamellar hepatocellular carcinoma. Am J Med Sci. 2009;338(6):522-4. https:/dx.doi.org/10.1097/MAJ.0b013e3181bccfb4 个Ver página 8

[13] Alsina AE, Franco E, Nakshabandi A, Albers C, Kemmer N, Berry AC, et al. Successful Liver Transplantation for Hyperammonemic Fibrolamellar Hepatocellular Carcinoma. ACG Case Reports J. 2016;3(1):e106. https:/dx.doi.org/10.14309/crj.2016.79个Ver página 8 